\title{
The massive eclipsing LMC Wolf-Rayet binary BAT99-129
}

\section{Orbital parameters, hydrogen content and spectroscopic characteristics}

\author{
C. Foellmi ${ }^{1}$, A. F. J. Moffat ${ }^{2}$, and S. V. Marchenko ${ }^{3}$
}

1 European Southern Observatory, 3107 Alonso de Cordova, Casilla 19001, Vitacura, Santiago, Chile e-mail: cfoellmi@eso.org

2 Département de physique, Université de Montréal, CP 6128, Succ. Centre-Ville, Montréal, QC, H3C 3J7, Canada

3 Department of Physics and Astronomy, Thompson Complex Central Wing, Western Kentucky University, Bowling Green, KY 42101-3576, USA

Received 18 August 2005 / Accepted 17 October 2005

\section{ABSTRACT}

BAT99-129 in the LMC is one among a handful of extra-galactic eclipsing Wolf-Rayet binaries known. We present blue, medium-resolution, phase-dependent NTT-EMMI spectra of this system that allow us to separate the spectra of the two components of the binary and to obtain a reliable orbital solution for both stars. We assign an O5V spectral type to the companion, and WN3(h)a to the Wolf-Rayet component. We discuss the spectroscopic characteristics of the system: luminosity ratio, radii, rotation velocities. We find a possible oversynchronous rotation velocity for the O star. Surprisingly, the extracted Wolf-Rayet spectrum clearly shows the presence of blueshifted absorption lines, similar to what has been found in all single hot WN stars in the SMC and some in the LMC. We also discuss the presence of such intrinsic lines in the context of hydrogen in SMC and LMC Wolf-Rayet stars, WR+O binary evolution and GRB progenitors. Altogether, BAT99 129 is the extragalactic counterpart of the well-known Galactic WR binary V444 Cygni.

Key words. binaries: eclipsing - stars: fundamental parameters - stars: Wolf-Rayet - stars: evolution - methods: data analysis stars: individual: BAT-129

\section{Introduction}

The Wolf-Rayet (WR) star BAT99-129 (a.k.a Brey 97, see Breysacher et al. 1999, hereafter BAT129) has been discovered to be a short-period eclipsing binary by Foellmi et al. (2003b). According to these authors, it consists of a nitrogenrich WN4 Wolf-Rayet component and a companion of unknown spectral-type. Along with BAT99-19 (Brey 16) in the LMC and HD 5980 in the SMC, BAT129 belongs to the very small group of extra-galactic eclipsing Wolf-Rayet binaries.

Absorption lines are visible in the spectrum of BAT129, especially in the blue part of the optical range $(\$ 4000 \AA)$, while WR emission lines strongly dominate everywhere else. The origin of these absorption lines is unclear: they are either produced by an O-type companion, and/or they originate in a WR wind, as has been found in all the single early-type $\mathrm{WN}$ (WNE) stars in the SMC and, to a lesser extent, in the LMC (Foellmi et al. 2003a,b; Foellmi 2004).

In the SMC about half of the WR population consists of hot, single, hydrogen-containing single WN3 and WN4 stars (classified WN3-4ha or (h)a). For such hot spectral types,

* Based on observations obtained at the New Technology Telescope, La Silla, Chile. significant amounts of hydrogen were not expected, since such hot WN stars are believed to have peeled off their outer H-rich layers by a very strong and optically thick wind, combined with internal convective mixing to expose core-processed material. Contrary to expectations, blueshifted absorption lines of $\mathrm{HI}$ and HeII were detected in the spectra of all single SMC WNE stars, and Foellmi et al. argued that they originate in a WR wind. Such stars were also found in the LMC, and more recently, in our Galaxy, in a region where the ambient metallicity may resemble that of the LMC: WR3, classified as WN3ha (Marchenko et al. 2004). Finally, two other Galactic, presumably single WNE stars clearly show hydrogen in their spectra: WR128 (WN4) and WR152 (WN3) (Crowther et al. 1995; Nugis \& Niedzielski 1995).

This paper examines a binary star. Hydrogen is already known to occur in the WR component of some binaries, e.g. in the 1.64-day eclipsing system CQ Cep (Marchenko et al. 1995). However, the authors classify the WR component as WN6, while Smith (1968) gives a spectral type WN7. These two spectral types seem to mostly describe very massive stars (see for instance the most massive star known: WR20a in our Galaxy, with two stars both of type WN6ha - Bonanos et al. 2004; Rauw et al. 2005) in a core hydrogen-burning 
Table 1. (1) Foellmi et al. (2003b). Summary of the 3 spectroscopic datasets obtained on BAT129.

\begin{tabular}{cccccc}
\hline \hline Dates & Observatory & Instrument & No. of spec. & Wavel. coverage & Res. power (2.6 pix resol.) \\
\hline 1998-2002 & See (1) & See (1) & 24 & $3800-6800 \AA$ & $\sim 900$ \\
2004, Sep. 22-25, Oct. 1st & NTT & EMMI & 21 & $3920-4380 \AA$ & 3500 \\
2005, Mar. 24-26 & NTT & EMMI & 12 & $3920-4380 \AA$ & 3500 \\
\hline
\end{tabular}

transition stage between the O Main Sequence and the core helium-burning WR stage (see also Foellmi et al. 2003b, for a detailed discussion of this important point). The question that remains to be addressed is: do we detect the presence of hydrogen in the early-type WN component of a short-period binary, and if yes, could such a presence be explained in the same way?

Previously, none of our spectra of BAT129 were of sufficient quality to detect absorption lines belonging to the companion and measure its radial-velocities (RVs), thus obtaining a reliable orbital solution for both components and helping to separate the two spectra, as well as to resolve the question of hydrogen intrinsic to the WN component.

We present in Sect. 2 new medium-resolution NTT-EMMI spectra of BAT129. We discuss in detail in Sect. 3 the iterative procedure we used to separate the spectra and find a reliable orbital solution. In Sect. 4, we assess the presence of hydrogen in the WR spectrum and examine all the results on this binary star: spectral types of both components, luminosity ratio, radii, mass-loss rates, masses. We discuss in Sect. 5 the relevance of the presence of hydrogen to the WR classification, $\mathrm{WR}+\mathrm{O}$ binary evolution and GRB progenitors. We draw conclusions in Sect. 6.

\section{Observations and data}

Our spectroscopic dataset of BAT129 is composed of three parts. The first part (binary discovery) was published by Foellmi et al. (2003b), to which the reader is referred for a detailed description. There one can also find the MACHO data used to obtain the light curve of BAT129. The second part consists of 21 medium resolution spectra spread over a few days, obtained with the ESO Multi-Mode Instrument (EMMI) at the New Technology Telescope (NTT) on La Silla, Chile. The third part is composed of 12 spectra with identical characteristics to the second dataset, but taken six months later. Table 1 summarizes the characteristics of each dataset.

For the new EMMI data, the Blue Medium-Resolution Spectroscopy mode (BLMD) was used, with Grating\#3 at a central wavelength of $4150 \AA$, providing a wavelength coverage of 3920-4380 $\AA$ and a dispersion of $0.45 \AA /$ pixel. The $1^{\prime \prime}$-wide slit provided a resolving power of 3500 (resolution of 2.6 pixels). The typical 30-min exposure resulted in $\mathrm{S} / \mathrm{N}$ ratio between 50 and 90 . The spectra were reduced using standard IRAF tasks and shifted to the heliocentric rest frame. Using continuum regions common to all EMMI spectra, the continuum was normalized to unity. The mean spectra of dataset 1 , and datasets 2 and 3 together, are shown in Fig. 1. It can be seen that the new spectra allow us to clearly resolve the emission and absorption components.
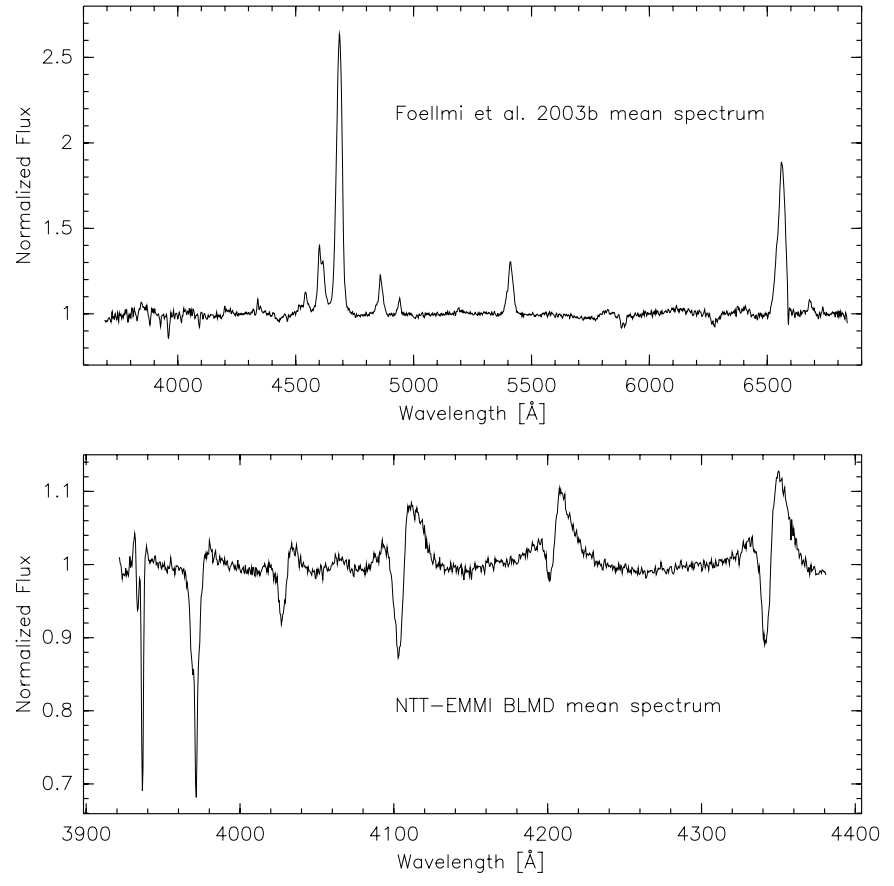

Fig. 1. Top panel: mean spectrum of data from Foellmi et al. (2003b). Bottom panel: EMMI mean spectrum. In this spectrum, the emissionline and absorption-line profiles are clearly resolved.

\section{Analysis}

We performed an iterative analysis on the EMMI spectra, that leads to a new and reliable orbital solution as well as separation of the individual spectra of the components. The general appearance of the extracted spectra, and therefore the shapes and strengths of features found in them, are very sensitive to the process of separation. We describe below in detail the analysis and the decisions we took in order to reach a reliable result.

\subsection{Photometric period, ephemeris and continuum correction}

Before computing the orbital phases for the EMMI spectra, we recalculated the ephemeris (period and zero point $E_{0}$ ) published by Foellmi et al. (2003b). The published values were based on the orbital solution obtained for RVs of emission lines only. In our new approach, we tried to minimize any influence of wind-wind collision (WWC) effects on the RVs. Furthermore, emission-line RVs of WR stars may not be a good indicator of the systemic RV, although orbital motion may not be a problem per se. This point is also discussed below.

A period search in the combined $\mathrm{R}$ and $\mathrm{B}$ MACHO data led us to a period of $2.7689 \pm 0.0002$ days, which is 




Fig. 2. MACHO light curve of BAT129, as described in Foellmi et al. (2003b), with the new value of $E_{0}$. MACHO instrumental magnitudes were transformed to obtain a mean magnitude outside the eclipse of 14.92 (Breysacher et al. 1999). The phases at which EMMI spectra were obtained are indicated by vertical lines. Full lines indicate spectra from the second dataset, whereas dashed lines indicate spectra from the third dataset.

equivalent to the published value. We note that with the Iband OGLE data (ID: LMC_SC18 122046/OGLE054148.68703531.0; see also Foellmi et al. 2003b), Wyrzykowski et al. (2003) obtain a value of 2.76895 days. To determine the zero point (i.e. time of primary minimum) $E_{0}$, we applied the bisector method on the deeper eclipse light-curve minimum, and obtained the following value: $E_{0}[\mathrm{HJD}]=2448843.8935 \pm$ 0.0007 . The resulting light curve folded in phase is shown in Fig. 2.

In order to be used in the separation procedure, the EMMI spectra must be corrected for the variable continuum level. We smoothed the lightcurve by taking mean values from 0.01 phase bins, converted magnitudes into fluxes and interpolated the smoothed lightcurve at the appropriate phases of the RV data. Then we computed the ratio between the flux at a given phase and the mean flux taken outside the eclipses, and appropriately normalized each spectrum by this ratio. The correction was at most $15 \%$. In that manner, the shapes (and equivalent widths) of the spectral lines do not depend on the variable continuum level.

\subsection{The "old" radial-velocities}

We also decided to re-measure the RVs of the first dataset, clearly affected by the traveling WWC emission details. New measurements were made using the bisector method on the strongest and best defined line: HeII $\lambda 4686^{1}$. The bottom level

\footnotetext{
1 We also assessed the HeII $\lambda 5411$ line, but discarded the measurements because of the relatively low $\mathrm{S} / \mathrm{N}$ ratio in $\sim 50 \%$ of the spectra.
}

for the bisectors was fixed at 1.125 (the continuum being at unity), in order to avoid blending problems. To minimize the influence of the emission component arising in the WWC zone, we fixed the top level at 1.5 , thus using the lower-third portion of the line. This proved to be quite reliable, since the results did not change significantly if we slightly modified these limits.

\subsection{The preliminary radial-velocities of the absorption profiles}

Before measuring RVs in the EMMI spectra, we used the two narrow interstellar Calcium lines H \& K at 3933 and $3968 \AA$, clearly visible in the EMMI spectra, to self-calibrate the RVs by co-aligning the IS features in the individual spectra, in $\mathrm{RV}$ space. The correction was at most $30 \mathrm{~km} \mathrm{~s}^{-1}$ for a given spectrum. We then removed these lines using Gaussian fitting, in order to avoid problems during the separation process (see below).

Simultaneous measurement of the emission and absorption profiles in the original EMMI spectra proved to be problematic. The uncertainties are introduced both by (a) the largeamplitude relative motion of the absorption and emission profiles, and (b) the traveling emission component produced in the WWC zone. We therefore chose at that point to measure the absorption profiles only, since they are relatively well-defined in each individual spectrum.

We measured positions of the absorption profiles via leastsquare fitting of Gaussian profiles (the IRAF task "splot") for the following lines: HeII $\lambda 4025, \mathrm{H} \delta$, HeII $\lambda 4200$ and $\mathrm{H} \gamma$. A weight of 0.5 was assigned to the relatively weaker absorptions at $\lambda 4025$ and $\lambda 4200$, compared to 1.0 for the rest. We thus combined these four lines into a single set of absorption-line $\mathrm{RVs}^{2}$.

\subsection{The first-iteration orbital solution}

Combination of the newly obtained RVs of the emission lines (dataset 1) with the RVs of the absorption lines in EMMI spectra leads to a complete set of spectroscopic orbital parameters. We applied the algorithm described in detail by Bertiau \& Grobben (1969) to obtain the solution. We fixed the period to the photometric value. Since the derived eccentricity did not significantly differ from zero, we subsequently adopted $e=0.0$ (but see below).

\footnotetext{
2 The situation is slightly more complicated since HeII $\lambda 4025$ is in fact a blend of the lines HeII $\lambda 4025.60$ and HeI $\lambda 4026.191$. Moreover, the Balmer lines $\mathrm{H} \delta$ and $\mathrm{H} \gamma$ are blended with HeII lines as well. In order to be perfectly precise, the positions of the profiles measured on the spectra should be compared to the rest wavelength of the corresponding lines. But in the case of blends, the contribution of each individual line is not known. We make here the reasonable hypothesis that the relative intensity contribution of one individual line to a given blend is not changing with time. Therefore, the comparison of a profile position to one rest wavelength is relevant and coherent. Thus the choice of one rest wavelength to another will only produce an offset in the absolute value of the RVs. At that point of our analysis, only relative RVs are meaningful. In general, except when specifically stated, the rest wavelength that is used is the one of the line specified explicitly in the text.
} 
Table 2. First iteration of orbital parameters of BAT129. The parameters published by Foellmi et al. (2003b) are also shown for comparison.

\begin{tabular}{lll}
\hline \hline Parameter & Foellmi et al. 2003b & This study \\
\hline$P($ days $)$ & $2.7687 \pm 0.0002$ & 2.7689 (fixed) \\
$K_{\mathrm{WR}}\left(\mathrm{km} \mathrm{s}^{-1}\right)$ & $398 \pm 7$ & $318 \pm 7$ \\
$K_{\mathrm{O}}\left(\mathrm{km} \mathrm{s}^{-1}\right)$ & - & $174 \pm 7$ \\
$e$ & $0.082 \pm 0.022$ & 0. (fixed) \\
$E_{0}(\mathrm{HJD}-2451100.0)$ & $820.47 \pm 0.09$ & $846.4468 \pm 0.009$ \\
$\gamma\left(\mathrm{km} \mathrm{s}^{-1}\right)$ & $245 \pm 15$ & $270 \pm 5$ \\
$\omega\left({ }^{\circ}\right)$ & $342 \pm 12$ & 270 (fixed) \\
$a_{\mathrm{WR}} \sin i\left(R_{\odot}\right)$ & - & $17.5 \pm 0.5$ \\
$a_{\mathrm{O}} \operatorname{sin~} i\left(R_{\odot}\right)$ & - & $9.5 \pm 0.6$ \\
$\sigma(\mathrm{O}-\mathrm{C})_{\mathrm{WR}}\left(\mathrm{km} \mathrm{s}^{-1}\right)$ & - & 39 \\
$\sigma(\mathrm{O}-\mathrm{C})_{\mathrm{O}}\left(\mathrm{km} \mathrm{s}^{-1}\right)$ & - & 17 \\
\hline
\end{tabular}

On the other hand, we allowed the emission-line RVs to exhibit a phase shift. Although our bisector measurements aimed to avoid (at least, substantially minimize) any WWC influence, it is still possible that these RVs may show residual WWC or other effects, therefore misrepresenting the genuine orbital motion (see the detailed discussion in Lewis et al. 1993). We allowed the phase shift to be an additional free parameter, introducing it in discrete steps of 0.001 days, from -0.2 to 0.2 days. We thus computed a series of orbital solutions, artificially shifting the time of emission-line RVs with these step values, and selecting the solution with the minimal rms deviation between the observed and calculated RVs. Table 2 shows the comparison between this solution and that previously published (Foellmi et al. 2003b).

Clearly, the newly obtained RVs provide much smaller $\mathrm{K}_{\mathrm{WR}}$ amplitude, by about $80 \mathrm{~km} \mathrm{~s}^{-1}$. This is due to the newer, better emission-line RVs from dataset 1 , and resulting higherquality fit of our RVs. The phase shift between the two sets of $\mathrm{RVs}$ is equal to 0.012 days, i.e. $17 \mathrm{~min}$, which is negligible. Moreover, the maximum RV difference between this solution and a solution with a null phase shift does not exceed $5 \mathrm{~km} \mathrm{~s}^{-1}$. The two sets of RVs can therefore be considered as being well in phase with each other.

\subsection{The first attempt at spectral separation}

We used the calculated RVs in an attempt to separate the spectral components in the EMMI data following the iterative method from Demers et al. (2002) (See also Marchenko et al. 1998). This method consists of the following steps, starting with the emission-line component:

1. shift the spectra (always in RV space) to the common rest frame of the WR star, using the (fitted) orbital RVs of emission lines;

2. combine the shifted spectra (possibly weighted by the individual S/N ratios), thus providing a "super WR spectrum", where the O-star features have been smeared out;
3. shift the resulting mean spectrum back to the original positions, one by one;

4. subtract the mean spectrum from the original spectra, thus obtaining a set of first-iteration, individual spectra of the O component;

5. using the RVs of absorption lines, shift these first-iteration absorption-line spectra to the common rest frame of the O star;

6. combine them (possibly weighted by individual $\mathrm{S} / \mathrm{N}$ ratios), thus providing a mean first-iteration absorption-line spectrum;

7. shift the mean absorption-line spectrum back to the original positions, one by one (either using the previously calculated RVs or estimating them via cross-correlation);

8. subtract this mean absorption spectrum from each of the original spectra, thus providing a better guess at the emission-line spectrum and finishing the iteration.

The process is repeated until a stable solution is found, usually after 3 to 5 iterations. The choices to be made, as well as sources of uncertainty in the separation procedure are the following:

- the weights during the combination of spectra;

- the possibility of editing by hand the combined spectra;

- the possibility to compute (via Cross-Correlation $=\mathrm{CC}$ ) new RVs in the currently extracted spectra, in each iteration, before shifting;

- the wavelength range used in the $\mathrm{CC}$;

- the reference spectrum used in the $\mathrm{CC}$;

- the spectra to be combined into a mean spectrum (all phases, undiscretionately or some only at specific phases?).

After many tests, we converged to the following: 1 . We decided not to use weights when combining the spectra in order not to favor any orbital phase, as we are dealing with a relatively small number of spectra. Most of our EMMI spectra have about the same $\mathrm{S} / \mathrm{N}$ ratio (between 50 and 90 ). This option proved to be of negligible influence. 2. Editing by hand the combined spectra proved to be important in order to force the process to converge rapidly. The editing was done in order to minimize the adverse alteration of the spectrum, by simply erasing absorption profiles from the WR spectrum and emission details (presumably related to the WWC effects) from the absorption spectrum. Tests reveal that this is absolutely necessary for adequate convergence. 3. When using the $\mathrm{CC}$ for computing new RVs before shifting, we chose the wavelength range 4000-4370 A, i.e. the spectral range comprising the 4 most important absorption lines: HeII $\lambda 4025, \mathrm{H} \delta$, HeII $\lambda 4200$ and $\mathrm{H} \gamma$. 4. The reference spectrum used in the $\mathrm{CC}$ computations was chosen to be the average spectrum from the previous iteration. 5. Finally, the spectra around phase 0.9 show very clear WWC emission line excess, and their profiles considerably influence the resulting mean spectrum. They are certainly not representative of an unperturbed WR spectrum, and their influence must be tested.

In order to test our separation process, we performed 4 different separations, called hereafter "loops". In the first two loops we used the fitted RVs from the orbital solution above 
during the first 2 iterations, then switching to the RVs computed with $\mathrm{CC}$ in 3 additional iterations. In the first loop we did not take the spectra at phase 0.9 when compiling the averages. Note that the 0.9-phase spectra were kept in the process, and we obtained extracted components at those phases, but they were not used during the combination of the spectra (steps 2 and 6). In the second loop we used all the spectra.

The two other loops used the RVs from the orbital solution only, during 5 iterations. No new RVs were computed with the CC method, thus final results being a "black-box" output of the iterative process, based on preliminary RVs of the first orbital solution. Again, we removed the phase 0.9 spectra when calculating the mean spectrum in the third loop, and treated all phases equally in the fourth loop.

The resulting spectra of the 4 loops were found to be very similar, at first sight. However, significant differences were found in the RVs that were computed from them. In fact, since we don't know a priori the correct shape of the spectrum of the WR and the O components, we have to find a way to discriminate between these 4 approaches. We used orbital solutions to achieve this.

\subsection{Exploring the parameter space of the orbital solutions}

As stated in the first orbital solution above, the emissionline RVs from dataset 1 , and the absorption-line RVs from EMMI spectra seem to be well in phase. To check this point again with the new absorption-line RVs on extracted O-star spectra, for each of the 4 different loops, we computed the RVs for the O spectra via CC in the wavelength range 4000-4370 ̊. Then, we looked for an orbital solution taking only these RVs and the emission-line RVs of dataset-1, allowing for a phase shift (i.e., again taking the solution with the smallest $\sigma(\mathrm{O}-\mathrm{C})$ ) as described above. We found 4 solutions in quite good agreement with the first orbital solution (see Sect. 3.4), with phase shifts ranging from -0.067 days $(-1.6 \mathrm{~h}, 2 \%$ of the period) to -0.012 days $(0.3 \mathrm{~h}, 0.4 \%$ of the period). This can be regarded as negligible, meaning that these EMMI absorption-line RVs obtained on extracted spectra are well in phase with the RVs of dataset-1, and give a stable overall result. These two sets of RVs can therefore be combined into a single set, and give us confidence on the results of the separation algorithm.

Second, we looked for orbital solutions taking the three sets of RVs, allowing for a phase shift of the emission-line RVs from the extracted WR spectra only (since the previous 2 datasets were found well in phase). Moreover, we assume here that the number of spectra we have and the coverage in phase they provide are good enough to find a reliable solution, without additional constraints such as a circular orbit. We assume that the best solution is the one which gives the minimal scatter between fitted and observed RVs, and the smallest value of the eccentricity and the smallest phase shift. For each of the 4 different loops, we looked for an orbital solution with a free phase shift between -0.2 and 0.2 day in steps of 0.001 day and with eccentricity as a free parameter.
Table 3. Adopted circular orbit of BAT129.

\begin{tabular}{ll}
\hline \hline Parameter & Circular orbit (adopted) \\
\hline$P($ days $)$ & 2.7689 (fixed) \\
$K_{\mathrm{WR}}\left(\mathrm{km} \mathrm{s}^{-1}\right)$ & $316 \pm 5$ \\
$K_{\mathrm{O}}\left(\mathrm{km} \mathrm{s}^{-1}\right)$ & $193 \pm 6$ \\
$e$ & 0. (fixed) \\
$E_{0}$ & $2451946.4274 \pm 0.0054$ \\
$\gamma\left(\mathrm{km} \mathrm{s}^{-1}\right)$ & $265 \pm 5$ \\
$a_{\mathrm{WR}} \sin i\left(R_{\odot}\right)$ & $17.3 \pm 0.4$ \\
$a_{\mathrm{O}} \sin i\left(R_{\odot}\right)$ & $10.6 \pm 0.5$ \\
$\sigma(\mathrm{O}-\mathrm{C})_{\mathrm{WR}}\left(\mathrm{km} \mathrm{s}^{-1}\right)$ & 34.0 \\
$\sigma(\mathrm{O}-\mathrm{C})_{\mathrm{O}}\left(\mathrm{km} \mathrm{s}^{-1}\right)$ & 13.4 \\
$M_{\mathrm{WR}} \sin ^{3} i\left(M_{\odot}\right)$ & $14 \pm 2$ \\
$M_{\mathrm{O}} \sin ^{3} i\left(M_{\odot}\right)$ & $23 \pm 2$ \\
\hline
\end{tabular}

For each of the 4 loops, we have two "best solutions", one with the smallest scatter $\sigma(\mathrm{O}-\mathrm{C})$ and one with the smallest eccentricity. It appears clearly in our results that the third loop (i.e. blind separation of 5 iterations without combining the 0.9 -phase spectra) provided both smallest values for $\sigma(\mathrm{O}-\mathrm{C})$ (of 36.6 and $39.2 \mathrm{~km} \mathrm{~s}^{-1}$, respectively), while all other solutions have a $\sigma(\mathrm{O}-\mathrm{C})$ above $40 \mathrm{~km} \mathrm{~s}^{-1}$. The set of spectra providing the orbital solutions of the best quality is therefore that of the third loop.

It is not too surprising that a "blind" separation loop produces the best results. Although 2 blind iterations (in loops 1 and 2) allow the process to converge rapidly to mean WR and $\mathrm{O}$ profiles, the next iterations with RVs computed from them are still very sensitive to any residuals.

When comparing the two "best" solutions of the third loop, it is clear that they are very similar, and can be considered as equivalent. All orbital parameters are identical within the errors, except for the phase shift. The solution with the smallest $\sigma$ has a phase shift of 0.127 days $(3.05 \mathrm{~h})$, while the one with the smallest eccentricity has a phase shift of 0.076 days $(1.8 \mathrm{~h})$. Given the fact that both solutions have a resulting eccentricity consistent with an almost circular orbit: 0.022 and $0.021 \pm$ 0.015 , respectively, we decided to choose the solution with the smallest $\sigma$ (i.e. the one with the 0.127 -day phase shift), and enforce a circular orbit for simplicity. Finally we adopted the circular orbit, summarized in Table 3, and shown in Fig. 3.

\subsection{The final separation}

Using the fitted RVs from the last orbital solution, we performed an ultimate "blind" (no CC measurements) separation with 5 iterations, with a limited set of spectra to combine (i.e., excluding phase 0.9) and no weights applied during the averaging. The extracted spectra are shown in Fig. 4.

It must be noted here that the shapes of the wings of absorption and emission lines are very sensitive to the adopted levels of local continuum. Given the broadness of the emission lines and their substantial shifts due to orbital motion, it is difficult to define adequately wide common line-free regions to be 


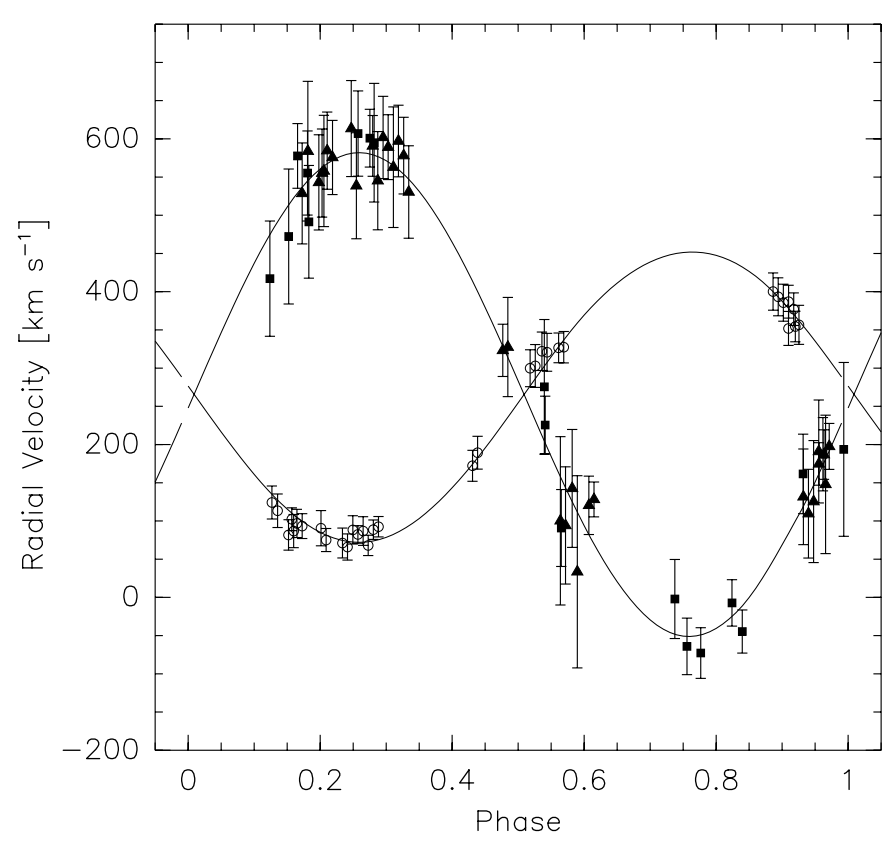

Fig. 3. Final (adopted) circular orbital solution including all 3 sets of RVs. Squares: RVs of emission lines (dataset 1). Triangles: RVs of emission lines (datasets 2 and 3). Circles: RVs of absorption lines (datasets 2 and 3).

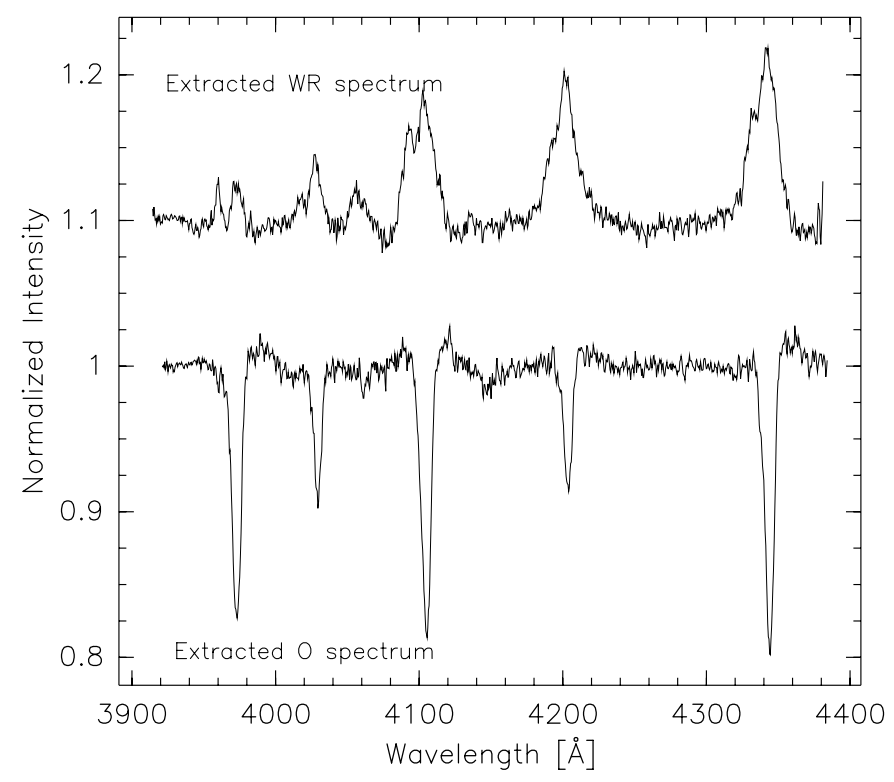

Fig. 4. Individual spectra of the WR and O components. The WR spectrum has been shifted vertically by 0.1 , while keeping the same scale.

used during continuum rectification. The most apparent consequence of a non-optimal continuum fit is the presence of lowintensity emission excesses in the wings of absorption lines in the O-star spectrum (see Fig. 4). To alleviate this problem, we repeated the whole separation procedure (i.e. from Sect. 3.2 to 3.7) twice, changing the approach to the continuum rectification. The continuum fits were done with the same polynomial, but once with a maximal number of available continuum regions and then with a minimal number of samples. The further steps were performed identically and led to the same sequence of decisions between the different orbital solutions. Finally, we chose the continuum rectification that provides the best (i.e. flattest) continuum on the final, extracted spectra, i.e. the one with the maximum continuum samples.

\section{Results}

We discuss here the results of the separation loops, i.e. the resulting separated spectra, their properties, and the orbital solution.

\subsection{The Wolf-Rayet absorption features}

One important detail can be seen in the separated WR spectra: absorption profiles on the blue sides of the emission lines. These features were always present in the final results of our separation tests. Hence, the presence of absorptions does not depend on our analysis of the continuum or on the editing "style" of the mean spectrum during the separation, nor does it depend on the choice of iterative sequence and the system of RVs we adopt while performing the separation.

Despite what is stated above, and in order to strengthen our confidence in the result of the separation, we measured the equivalent widths (EWs) of the 5 absorption lines (i.e. including this time $\mathrm{H} \epsilon$ ) seen in the $\mathrm{O}$ spectrum. If the separation process is reliable, we might expect that these EWs should not depend on the orbital phase. We also measured the EW of the 4 most prominent emission lines in the WR spectrum. The results are shown in Fig. 5. It appears that the EWs of the absorption lines in the individual extracted O-star spectra are roughly constant. No obvious periodic pattern or phase-locked variations are detectable in these EWs. Moreover, the EWs of the emission lines in the WR spectra are also relatively constant (see Fig. 5 - bottom figure).

Continuing to test the sensitivity of the overall appearance of the weak absorption components to details of the extraction algorithm, we attempted to construct an unperturbed WR spectrum. For that, we shifted the WR spectra to the same reference frame using the RVs of the accepted orbital solution. Then, we produced an average from the spectra around phases 0.4-0.6 (corresponding to inferior conjunction of the $\mathrm{O}$ star). The red side (up to $2000 \mathrm{~km} \mathrm{~s}^{-1}$ ) of the profiles was assumed to represent an unperturbed profile. Then, we combined the 3 spectra around phase 0.15 and considered the blue side of the average (down to $-2000 \mathrm{~km} \mathrm{~s}^{-1}$ ) as a fair representation of the unperturbed WR line profile. Then we merged the two sides for each line and subtracted the templates from the individual spectra. Unfortunately, the relatively low $\mathrm{S} / \mathrm{N}$ ratio of the spectra disallowed any further analysis of the absorption features, which were submerged in noise in all individual spectra.

A montage of the most prominent lines in the WR rest frame can also be used to understand more precisely what happens. The plots of $\mathrm{H} \delta$, HeII $\lambda 4200, \mathrm{H} \gamma$ (all coming from the EMMI data) and HeII $\lambda 4686$ (our dataset-1) are shown in Fig. 6. The montage of the HeII $\lambda 4686$ profiles shows very clearly the WWC excess-emission, traveling from the red side (around phase zero) when the $\mathrm{O}$ star is behind and the shock cone is pointing away from the observer, and reaching its bluemost position around phase 0.5 . On the montages of the three 

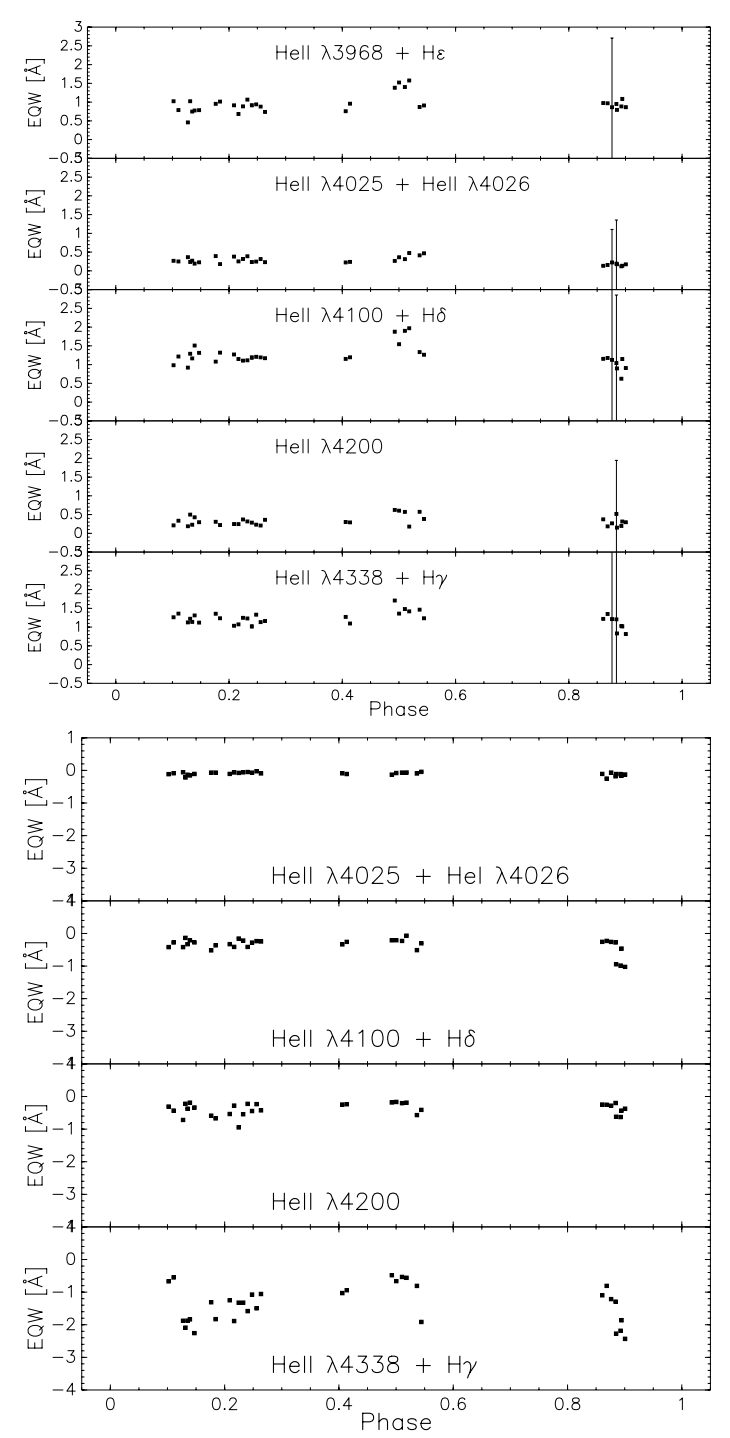

Fig. 5. Top figure: EW of absorption lines measured on the extracted O-star spectra. Bottom figure: EW of emission lines measured on the extracted WR spectra, including the WR absorption features. Note that panels of the two figures do not have the same vertical scale.

other lines, it can be seen that the absorption feature is most clearly seen between phases 0.15 and 0.25 . This is consistent with the fact that an excess emission is present in the red-center portion of the profile during that narrow phase interval. Then, when traveling to the blue, around phase 0.5 , this emission excess fills in the absorption feature, then retreating to the red side at phases around 0.9 and producing a profile with a pronounced redshifted bulge. As mentioned before, the latter caused problems during the separation process.

The ultimate test to check if the WR absorption lines do belong to the WR star is to measure the blueshifts of these absorptions, assessing both the blueshift on the mean WR spectrum (that is a measure of the separation between the WR absorptions and emission lines) and also blueshifts seen in individual lines (to prove that the blue-shifted absorption lines follow the WR orbital motion). To measure the blueshifts on individual spectra, we first shifted the WR spectra to the same frame using the orbital RVs, then combined them in orbital phase bins of 2 or 3 spectra in order to increase the $\mathrm{S} / \mathrm{N}$ ratio. A double Gaussian fit was performed on the 4 prominent lines cited above: HeII $\lambda 4025, \mathrm{H} \delta, \mathrm{HeII} \lambda 4200$ and $\mathrm{H} \gamma$, hence providing a position both for the absorption and the emission profile. The results are displayed in Fig. 7 (including only the measurements for which the fit provided a reasonable solution). The squares show the velocities of the absorption lines compared to the rest wavelength. The open circles show the velocities of the emission lines ${ }^{3}$.

Velocities of absorption and emission lines in Fig. 7 show a rough trend to be slightly in anti-phase. This is particularly clear for $\mathrm{H} \gamma$ where initially the RVs of emission and absorption are well separated, then become almost identical at phase 0.5 , and again move apart at phase 0.9 . Recall that all measurements are made in the WR rest-frame. Clearly, the excess emission coming from the WWC dominates the fits, apparently shifting the absorption line. However, the amplitude of the shifts of the absorption lines is much smaller than the corresponding O-star RVs, whose amplitude would be "enlarged" in the WR rest frame (the O-star motion is indicated by crosses in Fig. 7), and thus the absorption lines cannot belong to the $\mathrm{O}$ component. Disregarding the WWC-induced drift, the blueshift of the absorption lines can therefore be considered as constant relative to the rest wavelength, in the WR frame.

Finally, we took all the shifted WR spectra except those at phase 0.9 and combined them into a simple average, to increase the $\mathrm{S} / \mathrm{N}$ ratio. We then performed a double-Gaussian fit on the 4 prominent lines. The results are summarized in Table 4 , where the positions of the absorption and emission lines are compared to each other. The blueshift is clearly increasing with lower Balmer/Pickering number(s).

Altogether, these tests on the absorption lines seen in the WR spectrum allow us to claim that these blue-shifted lines belong to the WR star itself. Therefore, they are probably formed in the wind of the WR star, similarly to what has been seen in other single SMC and LMC stars (see Foellmi et al. 2003a, in particular).

\subsection{Spectral types of the WR and O stars}

Although they have a lower quality and the dataset is not homogeneous, we attempted to separate the orbital components with the spectra of dataset 1 , using a blind separation with 5 iterations, the orbital RVs and no editing. Thanks to the broader wavelength coverage and the lower resolution, we obtained a reasonably good O-star spectrum. The result is displayed in Fig. 8. A comparison of the mean O-star spectrum in BAT99 129 with standard spectra in the same (blue) range of Walborn \& Fitzpatrick (1990) leads to a type O5V. More precisely, the most frequently used features for determining the spectral class are HeII $\lambda 4541$ and HeI $\lambda 4471$. In our spectrum, the former is clearly stronger than the latter, pointing to a spectral type earlier than $\mathrm{O} 7$ (see close-up in

\footnotetext{
3 For the $\mathrm{H}+\mathrm{He}$ blends, the simple average of the rest wavelength has been taken. The important point here is to compare the velocities of the absorption and emission profiles together (see also previous footnote).
} 

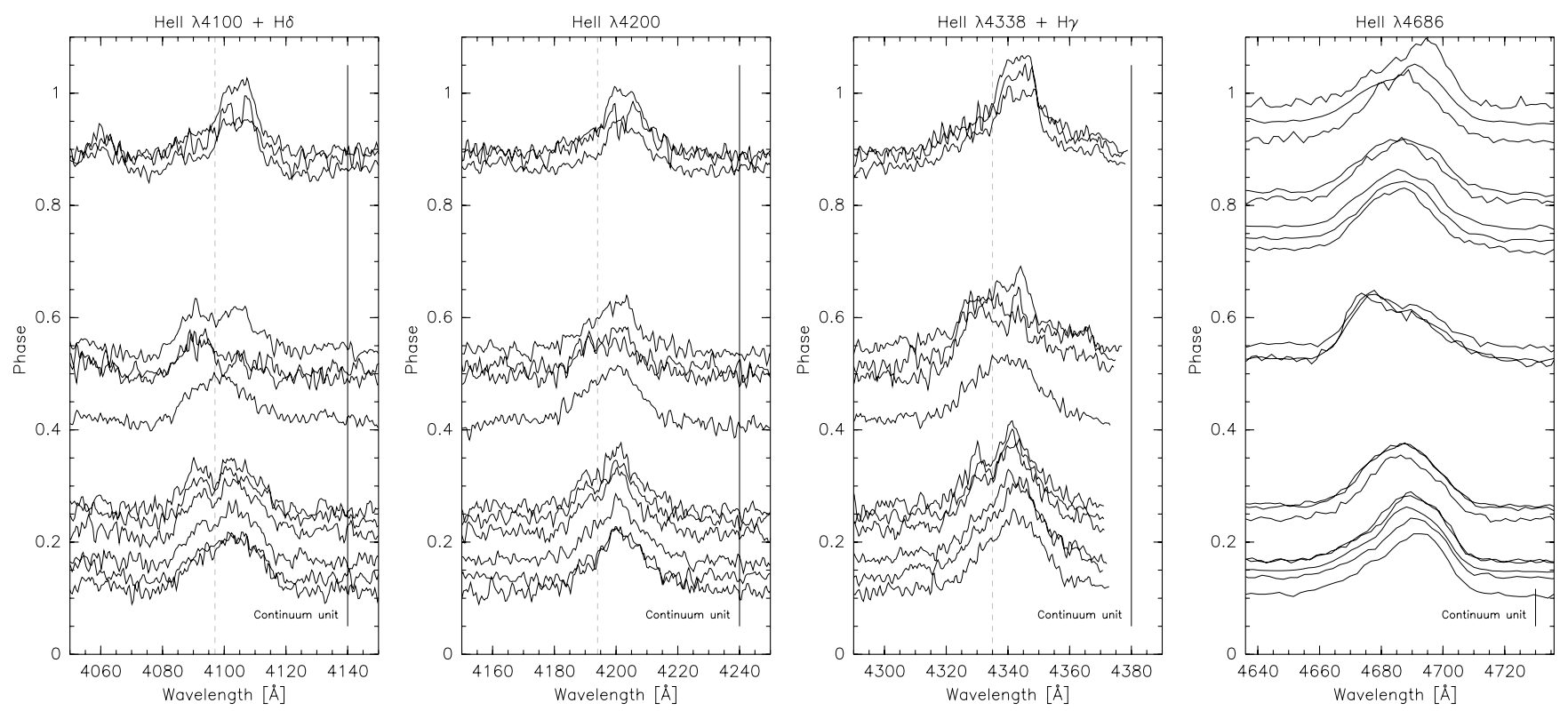

Fig. 6. From left to right: montage of $\mathrm{H} \delta$, HeII4200, $\mathrm{H} \gamma$ and HeII4686 from the WR component plus the WWC emission. All spectra were shifted to the WR rest-frame. For HeII4686 we used a reduction scale factor of 15. In the first 3 figures, the mean position of the absorption line is indicated with a dashed gray line.

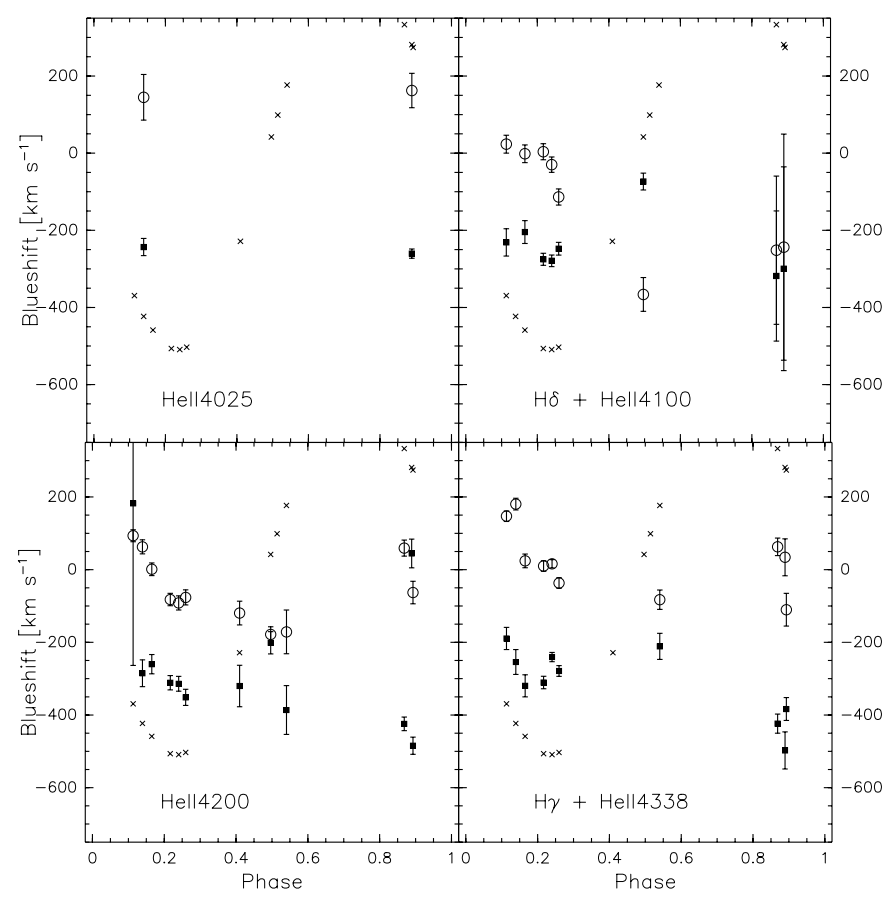

Fig. 7. Velocities of the WR absorption and emission lines obtained from the positions of the double Gaussian fits, versus orbital phase. The positions are compared to the rest wavelength of the corresponding line. For the $\mathrm{H}+\mathrm{He}$ blends, the simple average of the rest wavelengths has been taken. Filled squares: absorption lines. Open circles: emission lines. The crosses indicate the orbital RVs of the O-star in the WR frame.

Fig. 8). This is confirmed by the comparison of HeII $\lambda 4200$ and HeII + HeI $\lambda 4025$. We also measured the equivalent width of the lines HeII $\lambda 4541$ and HeI $\lambda 4471$ to compute their ratio. Following the spectral classification relation from Mathys (1988), we obtain $\log \left[E W_{4471} / E W_{4542}\right]=-0.48,-0.42$
Table 4. Blueshifts of WR absorption lines when compared to the positions of the emission lines.

\begin{tabular}{lr}
\hline \hline Line(s) & Blueshift $\left(\mathrm{km} \mathrm{s}^{-1}\right)$ \\
\hline HeI $\lambda 4025+$ HeII $\lambda 4026$ & $-86.3 \pm 18.3$ \\
H $\delta+$ HeII $\lambda 4100$ & $-161.8 \pm 12.5$ \\
HeII $\lambda 4200$ & $-228.7 \pm 11.5$ \\
H $\gamma+$ HeII $\lambda 4338$ & $-289.0 \pm 10.6$ \\
\hline
\end{tabular}

depending on the continuum position, with a preference for the first value. This corresponds to spectral types $\mathrm{O} 5$ and 05.5 respectively. Nonetheless, given the noisy nature of the spectrum, even a spectral type O6 cannot be excluded. As for the luminosity class, the extracted absorption lines are relatively broad, with no trace of SiIV beside $\mathrm{H} \delta$ on the higher-resolution EMMI spectra, hence broadly suggestive of a V-III luminosity class. Moreover, the line HeII $\lambda 4686$ in Fig. 8 is (particularly) strong in absorption, and this defines a luminosity class V.

The WN spectrum resulting from the separation loop can be compared to the criteria of Smith et al. (1996). Using the peak values, we obtain a ratio for HeII $\lambda 5411 / \mathrm{HeI} \lambda 5876=16.1$. This points towards a WN3 subclass, that is confirmed by the peak ratios of NV $\lambda 4604$ and NIII $\lambda 4640$, since the latter is very weak. The practical absence of the CIV $\lambda 5808$ line makes the result definitive: WN3. Finally, we measured the Pickering emission-line decrement. Using the description of Smith et al. (1996), the heights of $\mathrm{H}+\mathrm{He}$ lines clearly exceed the line drawn between pure Helium lines. Results are shown in Fig. 9. Moreover, the two hydrogen criteria of Smith et al. are equal to 0.22 and 0.24 , respectively. These are probably lower limits since the H-containing blends are more suppressed by stronger absorption components than the pure $\mathrm{He}$ lines. Combined with the presence of $\mathrm{H}$-absorption lines, 


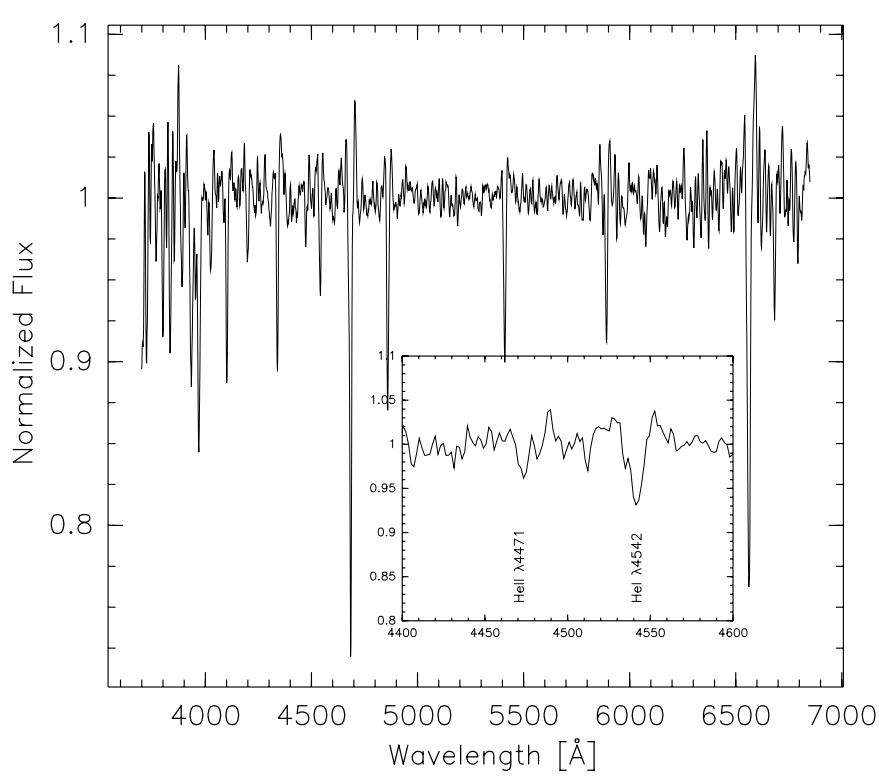

Fig. 8. O-star spectrum obtained by a separation process on the spectra of dataset 1 . The inset shows the region around the two lines HeII $\lambda 4471$ and HeI $\lambda 4542$ used for classification.

this leads to the moderate presence of hydrogen and a final type of WN3(h)a. Therefore, BAT129 appears to be an early-type hydrogen-containing WN star with a dwarf O5 companion.

\subsection{Luminosity ratio}

Using the methods described in Cherepashchuk et al. (1995) we derived the (visual) luminosity ratio $q \equiv L_{\mathrm{WR}} / L_{\mathrm{O}}$. To achieve a meaningful ratio, we combined EMMI spectra between phases 0.11 and 0.25 , i.e. outside the eclipses phases (the spectra of dataset 1 were not of sufficient quality and resolution for this). The classical method by Beals (1944) provides an upper limit, and the new method by Cherepashchuk et al. (1995) gives the "correct" value (i.e. corrected by the fact that the absorption and emission lines are superimposed on each other). The determination of the luminosity ratio is made by comparing the equivalent widths (EW) of O-star absorption lines both in the original mean WR+O spectrum and the "pure" O-star mean spectrum. We measured the EWs on the following lines, by Gaussian fitting: $\mathrm{H} \epsilon$, HeII $\lambda 4025, \mathrm{H} \delta+\mathrm{HeII} \lambda 4100$, HeII $\lambda 4200$ and $\mathrm{H} \gamma+\mathrm{HeII} \lambda 4338$.

Two problems arise here. First, the absorption lines belonging to the WR star in the WR+O spectrum must be removed. To account for this, we corrected the EW of absorption lines in the original $\mathrm{WR}+\mathrm{O}$ spectra by measuring the EW of the WR absorption lines in the extracted WR spectra:

$E W_{\text {bin }} \equiv E W_{\mathrm{WR}+\mathrm{O}}-E W_{\mathrm{abs}, \mathrm{WR}}$.

Also:

$q=\frac{E W_{\text {single }}}{E W_{\text {bin }} \cdot L C R}-1$,

where $L C R$ stands for "Line-to-continuum ratio" and is the measure of the line strength to the continuum level (i.e. peak

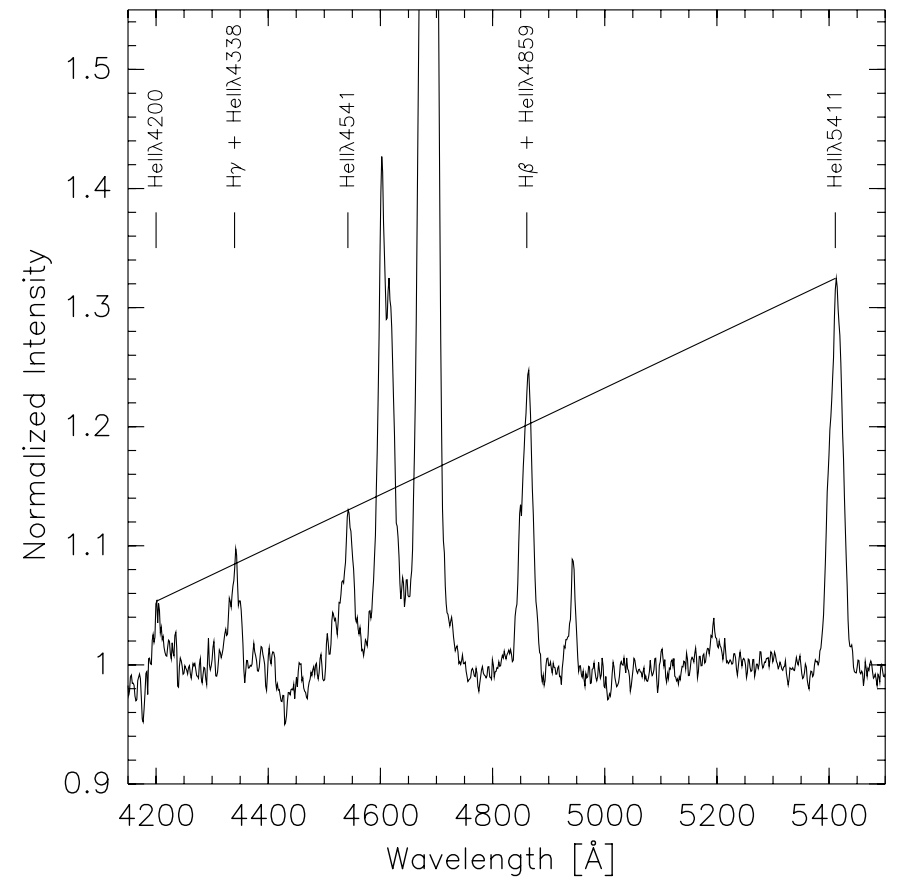

Fig. 9. WR spectrum obtained by a separation process on the spectra of dataset 1 . The line is used to show the Pickering emission-line decrement, leading to a moderate (h) presence of hydrogen in the WN spectral type.

value) in the WR+O spectrum (see Cherepashchuk et al. 1995, for the detailed equations).

The second problem is to compare our values of $E W_{\text {single }}$ to that of single $\mathrm{O}$ stars. We first measured the EW on the extracted O-star spectrum. Then, comparing our results with the values of Conti \& Frost (1977) for the lines HeII 14025 , HeII $\lambda 4200$ and $\mathrm{H} \gamma$, it seems that our values for the helium lines are overestimated, while that of $\mathrm{H} \gamma$ is underestimated (by about 0.3 and $0.6 \AA$ respectively). Taking lower values for the Helium lines give inconsistent results, since the EW on the combined binary spectrum would be larger than that of the single spectrum. Taking the upper value for $\mathrm{H} \gamma$ would actually correct the result of this specific line to a level comparable to that of the other lines. Although not fully reliable (since the result depends heavily on the output of a complicated separation process), we consider that our measurements on the extracted spectra are nevertheless globally consistent.

Assuming of course that there is no third-light contamination, and taking these values with care, we obtain the following mean luminosity ratios: $0.39 \pm 0.18$ and $0.28 \pm 0.20$, respectively with the two methods. In the following, we assume a luminosity ratio of 0.3 .

\subsection{The O-star characteristics}

Given the spectral type we assigned to the $\mathrm{O}$ star, the temperature-spectral type relation published by Massey et al. (2005) in their detailed study of LMC O stars, implies an effective temperature of about $40 \mathrm{kK}$, interpolating roughly the values between the SMC and the Milky Way relations. Similarly, using the scale for a solar metallicity and for galactic dwarf 
Table 5. Projected rotational velocities inferred from the O-star absorption lines, assuming that the broadening of the lines is due only to Doppler broadening. Values have been corrected by the 2.6-pixel resolution.

\begin{tabular}{lll}
\hline \hline Line & $F W H M(\AA)$ & Velocity $\left(\mathrm{km} \mathrm{s}^{-1}\right)$ \\
\hline $\mathrm{H} \epsilon+$ HeII $\lambda 3968$ & 6.72 & 195 \\
HeII $\lambda 4025+$ HeI $\lambda 4026$ & 5.51 & 158 \\
H $\delta+$ HeII $\lambda 4100$ & 7.43 & 209 \\
HeII $\lambda 4200$ & 6.61 & 181 \\
H $\gamma+$ HeII $\lambda 4338$ & 7.19 & 191 \\
\hline
\end{tabular}

stars by Martins et al. (2005) implies $T_{\text {eff }}=41 \mathrm{kK}$ for a spectral type O5V. However, these latter authors emphasize metallicity effects which provide higher temperature at lower metallicity. If we consider a shift of about one spectral subclass, this gives (for an O4V star): $T_{\text {eff }}=43 \mathrm{kK}$. For such a temperature, the luminosity of the $\mathrm{O}$ star is $\log \left(L / L_{\odot}\right)=5.49$. Let's assume this value of $L$ for the secondary star in BAT129.

One of the stars in the sample of Massey et al. (2005), namely LH 58-496, has the same spectral type (O5V) and thus the assumed luminosity $\left(\log \left(L / L_{\odot}\right)=5.49\right)$ of the $\mathrm{O}$ star in BAT129. The radius of the star quoted by the authors is $10.5 R_{\odot}$. Following Eggleton (1983), this value is slightly smaller than that of the Roche radius of the O star in BAT129 $\left(11.8 R_{\odot}\right)$. Moreover, the evolutionary mass of LH 58-496 is $40 M_{\odot}$, compared to the measured Keplerian mass of BAT129: $M_{\mathrm{O}} \sin ^{3} i=23 M_{\odot}$ (see Table 3$)$. To reach a value of $40 M_{\odot}$ for BAT129, the inclination angle of BAT129 should be 57 degrees. Given the strong eclipsing nature of BAT129, this value of the inclination should be considered as a minimum estimate. Considering the large radius of the $\mathrm{O} 5$ star, the secondary component of BAT129 well may be an O6, with a smaller radius.

In their sample, Massey et al. (2005) have an O6.5V star, $\mathrm{AV} 446$ (in the SMC though). It has a temperature of $T_{\mathrm{eff}}=$ $41.0 \mathrm{kK}$, an evolutionary mass of $33 M_{\odot}$, and a radius of $8.8 R_{\odot}$ (i.e. $75 \%$ of the $\mathrm{O}$-star Roche radius). These values are also quite consistent with the estimates we draw on the secondary star of BAT129.

Given the short period, it is likely that the system is synchronized. If so, then with a radius of $8.8 R_{\odot}$, the equatorial velocity becomes $161 \mathrm{~km} \mathrm{~s}^{-1}$. However, the $F W H M$ measured on the extracted $\mathrm{O}$-star spectrum gives a projected rotational velocity slightly larger by about 20 to $40 \mathrm{~km}^{-1}$, as shown in Table 5 (assuming that the dominant broadening cause is the Doppler effect). On the other hand, these values are very close to the synchronized velocity of the star if it had a radius of $10.5 R_{\odot}\left(192 \mathrm{~km}^{-1}\right)$. Oversynchronicity is already known to occur in some WR+O binaries, such as V444 Cyg (Marchenko et al. 1994) and CQ Cep (Marchenko et al. 1995).

\subsection{The WR-star characteristics}

The visual apparent magnitude of BAT129 quoted by Breysacher et al. (1999) is 14.92, refering to a work by Rousseau et al. (1978). Assuming a distance modulus of 18.5 for the LMC (van den Bergh 2000) and IS visual extinction of $A \mathrm{v}=0.4$ (i.e. $3.1 * 0.13$, see Massey 2003; Conti \& Morris 1990), the absolute visual magnitude of the system is -3.98 , i.e. fainter than the magnitude of the single $06.5 \mathrm{~V}$ star mentioned above $\left(M_{V}=-4.7\right.$, Massey et al. 2005). The Johnson magnitude of BAT129 given by Feitzinger \& Isserstedt (1983) is 14.68 , i.e. $M_{V}=-4.2$, which is still fainter than the O star value ${ }^{4}$. Two obvious reasons can explain the above discrepancy. First, the measured magnitude of the system is too faint because the eclipses were not accounted for. This effect is unlikely to resolve the discrepancy, since it is smaller than 0.2 mag (see Fig. 2). Second, the spectral type inferred from our extraction process is wrong and a cooler spectral type must actually be assigned to the O star in BAT129. This second possibility is more plausible, although we have no definite means to verify it.

We can nonetheless compare the properties of the WR star in BAT129 with LMC WN stars studied by Hamann \& Koesterke (2000). Taking our luminosity ratio of 0.3 , the WR star has a luminosity of $\log \left(L / L_{\odot}\right)=4.97$, and $M_{V}=$ -3.34 . This absolute magnitude is nonetheless close to the average value for the Galactic WN3 stars (van der Hucht 2001), and slightly fainter than that of WR3 in our Galaxy $\left(M_{V}=\right.$ -3.7, Marchenko et al. 2004)

Given the luminosity of the WR star in BAT129 and its absolute visual magnitude, the most similar star in the selected subsample of Hamann \& Koesterke (2000) is BAT99 63 (Brey 52): it has a spectral type WN4, a temperature of $71 \mathrm{kK}$, $50 \%$ hydrogen (by mass), a radius $R_{*}$ (i.e. at a Rosseland optical depth of 20) of $4.7 R_{\odot}$ and a mass-loss rate $\log \left(\dot{M} / M_{\odot}\right)=$ -5.2 . This mass-loss rate value is in agreement with the recent theoretical value computed by Vink \& de Koter (2005), for the metallicity of the LMC: $-5.26,-5.49$ for $\beta$ (exponent of the wind velocity law) equal to 1 and 3 , respectively. In the MACHO light curve, spanning more than 10 years, we have also searched for any significant period changes. We have split the lightcurve into 10 equally long bins, and performed a Fourier analysis on each of them. Note that given the number of datapoints and their spread in time, the maximum number of bins allowing a precise analysis is not much larger than 10 . We found no significant period changes, with a precision of 0.003 days over the whole timespan. However, this precision does not allow us to put any meaningful constraint on the WR mass-loss rate. Comparing the Keplerian mass of the WR component in BAT129 $\left(M_{1} \sin ^{3} i=14 M_{\odot}\right)$ to the $a c$ tual mass of BAT63 quoted by Hamann \& Koesterke (2000) $22 M_{\odot}$ - the inclination angle in BAT129 must be 59 degrees, which is equivalent to the estimate drawn from the $\mathrm{O}$ star.

For the moment, let us assume that the WR star in BAT129 has followed a pure single-star evolution. The WR star must have had an initial mass above that of the current secondary star, i.e., at least $35 M_{\odot}$. On the other hand, if we assume that the WR star is, say, in the middle of its eWNL phase (i.e. its h-rich WN phase, see below), we can use the mass

\footnotetext{
4 We were not able to compute the correct MACHO magnitude, following Eqs. (1) and (2) of Alcock et al. (1999), since some coefficient values were not accessible on the Internet, at the time of writing.
} 
loss rate quoted above to estimate the amount of mass lost by the star during the WR stage. The duration of the Heliumburning phase of a $40 M_{\odot}$ star, following the models of rotating WR stars at $Z=0.008$ of Meynet \& Maeder (2005), is 0.550 million years. Assuming the WR star in BAT129 is well described by these models, this means that the star has lost slightly less than $2 M_{\odot}$ during the WR phase. The $60 M_{\odot}$ model would imply an even lower value, but even the $30 M_{\odot}$ model does not give a value larger than $3 M_{\odot}$ lost by the WR. Taking the slightly smaller mass-loss rate of WR3 in our Galaxy $\left(\log \left(\dot{M} / M_{\odot}\right)=-5.62\right.$, Marchenko et al. 2004) of course does not resolve the situation. This means that the mass-loss rate quoted above is certainly not valid for the WR star in BAT129 as a single star, since the estimated mass lost is too small by a factor $5\left(2-3 M_{\odot}\right.$ compared to at least $10 M_{\odot}$ from its initial mass).

With the assumption that BAT129 is a non-interacting system and that no RLOF occurred in its past evolution, some of the parameters of the WR star and its companion can be relatively well explained by single-star evolution. However, the combination of the possible oversynchronous rotation of the $\mathrm{O}$ star, the very short period of the system and the theoretical single-star mass loss rate clearly too small seem to point toward an interacting scenario for BAT129, although the presence of hydrogen in the WR star might be contradictory at first sight. This is discussed in the next section in a broader perspective of the WN evolution with hydrogen.

\section{Discussion}

It appears that weak absorption features are superposed on the emission line profiles in the extracted WR spectra. We have shown that the features are moving with the WR star. These absorption features are blueshifted relative the main emission profiles, and the blue shift seems to increase in the lines with progressively lower Pickering (or Balmer) number. Therefore, we are tempted to conclude that these absorption features are identical to the intrinsic absorption lines seen in many earlytype WN Magellanic Cloud stars and WR3 in our Galaxy. However, for the first time we detect them in a short-period binary. The presence of hydrogen in an early-type WN star in a binary has important consequences that are discussed below.

\subsection{Wolf-Rayet star classification}

The presence of hydrogen in WN stars is relevant for their classification and from the point of view of their evolutionary status. Most of the late-type WN stars (WNL) have hydrogen in their winds, as they are believed to be the first WR stage after the main-sequence and/or a possible transition stage. However, a significant number of exceptions exist to this rule. Similarly, most of the early-type WN stars (WNE) are hydrogen-poor, or hydrogen-free, but again, with a significant number of exceptions. For some reasons, that are likely to be due to rotation, the exceptions are more numerous in the SMC. Five out of $11 \mathrm{WN}$ stars in the SMC have a very hot spectral type (WN4, WN3) and present signatures of a large amount of hydrogen (ratio $\mathrm{H} / \mathrm{He}$ by number $\sim 1$, P. Crowther, private communication). Therefore, the ionization subclass (i.e. the temperature) is not the decisive criterion to distinguish between late and early-type WN stars. This is the reason why Foellmi et al. (2003b) proposed a complementary and evolutionary classification for WN stars (recognizable by putting an "e" in front of the class) which is based on the hydrogen content. The main eWNL/eWNE distinction is now based on the amount of hydrogen. To discriminate the h-rich from the h-free stars, the "b" (for "broad" lines) classification criterion of the classification scheme of Smith et al. (1996) can be used. As a matter of fact, Hamann et al. (1995) have shown that the width of the emission lines is directly connected to the hydrogen content (see also Smith \& Maeder 1998). Moreover, since a clear anti-correlation between the "b" and " $\mathrm{h} /(\mathrm{h})$ " spectral notations of WN stars is observed in LMC stars (Foellmi et al. 2003b) - an effect already mentioned by Smith et al. (1996) - the "b" criterion is an "antipode" of the "h" criterion.

This classification was meant for single stars, since the hydrogen content in WN stars in binaries was not known. With BAT129, we can extend the applicability of this classification. It is rather natural to think that the same causes produce the same effects: when the lines are narrow, it means that hydrogen is present in the atmosphere of a WR star, and in some cases where the amount of $\mathrm{H}$ is substantial, an alterning Pickering emission-line serie and/or absorption lines may be visible in the optical spectrum. Moreover, we note that this classification implies that the SMC contains virtually no eWNE stars, but only eWNL stars (and one WC/WO binary). This fact is well reproduced by the recent models of rotating WR stars at low metallicity of Meynet \& Maeder (2005, see their Fig. 9).

\subsection{Evolution of $W R+O$ binaries}

A simple estimate of the minimum period of BAT129 allows us to show that the system was certainly interacting in the past. Let us assume a ZAMS mass of the O star similar to the estimated mass above (say, $35 M_{\odot}$, i.e. the star is assumed to have lost no mass), and the corresponding Main Sequence (MS) radius: $\sim 10 R_{\odot}$ (see e.g. Martins et al. 2005, their Table 1). Assuming that the present WR star had an initial mass of, say, $45 M_{\odot}$, and became a WR star by simple wind mass-loss, its maximum radius during the MS phase is the radius of a $45 M_{\odot}$ MS supergiant: $\sim 20 R_{\odot}$ (i.e. not considering a red supergiant nor a Luminous Blue Variable phase; see Martins et al. 2005, their Table 3 for the radius value). Assuming that the minimum distance between the two stars is at least the sum of their radii $\left(d_{\min }>30 R_{\odot}\right)$, and using Kepler's third law, the minimum period of the system is 2.2 days, which is very similar to the current period. Therefore, given the extreme example considered here, the widening of the orbit from 2.2 to 2.7 days caused by a simple mass-loss by the stellar wind during the WR phase of the primary component can be ruled out. This implies that to obtain the present short period, the system must have gone through an interacting phase that shrank the orbit.

Furthermore, it is very interesting to compare the actual parameters of BAT129 with the latest models of binary star evolution of Petrovic et al. (2005a). The models of binary stars 
that lead to $\mathrm{WR}+\mathrm{O}$ systems show that Roche-lobe Overflow (RLOF) and mass transfer occur for short-period systems. We can distinguish Case A mass transfer, that starts during the core hydrogen-burning phase of the primary, and Case B mass transfer starting during the shell hydrogen-burning phase. As emphasized by Petrovic et al. (2005a), the net effect of a Case A (that also contains an intermediate Case $\mathrm{AB}$ mass transfer episode) or a Case B scenario is the increase of the period. If such mass transfer occurred in the past in BAT129, this means that the initial period would have been even smaller than about 2 days, which is very unlikely. Therefore, following Petrovic and collaborators, the evolution of BAT129 should be considered as the probable result of contact evolution.

Wellstein et al. (2001) have studied the formation of massive contact binaries. Unfortunately, among the 74 models they have computed, none of their models actually include primary stars more massive than $25 M_{\odot}$. If we nonetheless consider globally their results, their models also lead to an increase of the period after the RLOF (whatever its kind). We note here that an oversynchronous rotation velocity of the secondary star is known to occur in other $\mathrm{WR}+\mathrm{O}$ systems, among which is the famous Galactic system V444 Cyg (Marchenko et al. 1994), WR127 (Massey 1981) and CQ Cep (Marchenko et al. 1995). Interestingly, while CQ Cep is supposed to have experienced a contact phase, this is not the case for V444 Cyg, which is relatively well explained by a stable mass transfer scenario, according to Petrovic et al. (2005a). The uncertainty on the radius of the WR star in BAT129 is unfortunately too large to allow a conclusive discussion on this phenomenon related to the interaction of the two stars.

Altogether, it seems likely that BAT129 followed an interacting (possibly contact) evolution. A detailed analysis of the abundances of the $\mathrm{O}$ star could possibly help to probe the question of whether the companion star has accreted mass from the primary, and how much. We have obtained very high S/N FORS1 spectra at the VLT of BAT129 that could possibly allow such analysis. This is left for a forthcoming paper.

\subsection{Gamma-ray burst progenitors}

In this section we try to consider the binary star BAT129 or its WR component alone as "test particles" for the scenario of formation of Gamma-ray bursters (GRB).

Although WR stars are believed to be the progenitors of Type Ib/c supernovae associated with long-duration GRBs (Galama et al. 1998), quite successfully described by the "collapsar" model (MacFadyen \& Woosley 1999), it is still very difficult to answer the question: what kind of WR stars are the best candidates for a GRB? Petrovic et al. (2005b) try to address this issue from a theoretical point of view. They emphasize that three essential ingredients are needed to produce a GRB: a massive core to form a black hole (BH), sufficient angular momentum to form an accretion disc around the $\mathrm{BH}$ with bipolar jets, and loss of hydrogen envelope to let bipolar jets reach the star surface. They show that both single and binary non-magnetic WR stars can retain enough angular momentum for a GRB to form. Hence the question remains: are binaries more favorable GRB progenitors than single stars?

The recent models of single rotating WR stars at different metallicities of Meynet \& Maeder (2005) show 3 important things. (1) Final surface velocity at low metallicity is higher than at high metallicity, a fact that is explained as a consequence of the lower mass loss rates at lower metallicity. Therefore, low metallicity galaxies such as the SMC and the LMC are better locations to find fast rotating WR stars at the end of the He burning phase, in agreement with the results of Le Floc'h et al. (2003) who show that GRB occur mostly in blue sub-luminous galaxies (see also Prochaska et al. 2004). (2) At a given metallicity the velocities obtained at the end of the He-burning phase are larger for smaller initial mass stars. (3) The last point is that most of the models of Meynet \& Maeder (2005) at low metallicity have enough specific angular momentum in their core to be good candidates for collapsar models. Again we can ask: are binary stars better GRB candidates than single stars, even if single star WR evolution provides the necessary conditions for such an event?

How does BAT129 fit in this picture? While the metallicity is that of the LMC, and the combination of the very early spectral type and the presence of hydrogen is probably a sign of rapid rotation (Foellmi et al. 2003a; Foellmi 2004), the main problem is to know what was really the initial mass of the WR star. If it had followed a single-star evolution, it is likely that it was initially a lower-mass star (say with an initial mass between 20 and $40 M_{\odot}$ ). This is consistent with its large rotational velocity (see again the corresponding model of Meynet $\&$ Maeder 2005), and the clear presence of hydrogen. However, we have shown above that the characteristics of BAT129 as a binary make it a good candidate for an interacting binary, possibly with a contact phase. We can therefore ask: could the initial mass of the WR star be very large (say above $50 M_{\odot}$ ), and most of it has been lost in the interaction of the binary? Could binary evolution produce such a WR+O system where the WR star looks like its SMC counterparts? If the WR star lost a large amount of mass and the secondary did not accrete all the material, it is likely that a nebula should be seen around BAT129. We find however no clear nebular lines in our spectra.

Tutukov \& Cherepashchuk (2004) also mention that the creation of a $\mathrm{BH}$ and the generation of a GRB associated with it is most probable in the closest massive binary systems (not only $\mathrm{WR}+\mathrm{O}$ but also $\mathrm{WR}+$ [compact companion] binaries), since the presence of a close companion limits the rotational angular velocity of the WR core to the orbital angular velocity of the binary system. Very short period WR+O binaries formed through a common envelope phase, as BAT129 could be, is very relevant in this picture (see also the models by Wellstein \& Langer 1999). Although not a definitive argument, we note that the rarity of very close WR binary systems might help the frequencies of GRBs and fast rotating WR core collapses to agree (see e.g. Postnov \& Cherepashchuk 2001).

The point of GRB progenitors is further investigated by Hirschi et al. (2005) for single star evolution. They note in particular that angular momentum at the end of the He-burning is a good approximation of the angular momentum contained in the core at the collapse. This is an important point for 
observational tests, since even the pre-core collapse evolution phases are rather short and unlikely to be extensively observed. The authors also give a rough estimate of the initial mass range to form a $\mathrm{BH}$, that is between 20 and $60 M_{\odot}$. But forming a $\mathrm{BH}$ is not sufficient to produce a GRB; the angular momentum must be large enough to build up an accretion disk that will power bipolar jets. Interestingly, they show that WR stars of the WO subtype are the most probable GRB progenitors, since they are formed at low metallicity, and always form a BH (see also Postnov \& Cherepashchuk 2001). However, the initial mass of a WO star is estimated to be $\gtrsim 50 M_{\odot}$.

All the above considerations lead to the reasonable conclusion that GRB progenitors are certainly a special trade-off of the different parameters of WR evolution. Some things seem however to be clear. The metallicity should be low, and the initial mass of the WR star not too high to avoid any strong mass loss, and in particular any Luminous Blue Variable (LBV) event (see Meynet $\&$ Maeder 2005, for limiting masses of the 3 main evolution scenarii of WR stars). It is interesting to note that in the models of Meynet \& Maeder (2005) at SMC metallicity $(Z=0.004)$, only the $60 M_{\odot}$ model has clearly a higher rotational velocity at the end of He burning, while the lowest initial mass models (30 and $40 M_{\odot}$ ) at LMC metallicity $(Z=0.008)$ have the largest rotational velocity. We may wonder whether only single WO stars at the lowest metallicity produce GRBs, while lower mass WR stars do so at slightly higher metallicity. In that context, the unique WO binary star of the SMC (WR8) deserves probably much more attention (see e.g. Bartzakos et al. 2001).

One may also want to discuss the growing amount of evidence that hydrogen is clearly detected in GRB afterglows. This has been first discussed by Rigon et al. (2003) for supernova SN 1999E possibly associated with the BATSE event of GRB 980910. Recently, Starling et al. (2005) found the clear signature of hydrogen in the spectrum of the early afterglow of GRB 021004. Finally, van Marle et al. (2005) have made evolutionary models of circumstellar material around a massive star that produces a GRB. It is therefore relevant to search for the characteristics of hydrogen in WN stars. As suggested by the analysis of WR3 in our Galaxy (Marchenko et al. 2004), the absorption lines form relatively close to the stellar core. It is likely that the absorbing material is then gradually accelerated to the terminal wind velocity. Since we have shown that it is not unusual to see a significant amount of hydrogen in single and binary WN stars at low metallicity, it might be not too surprising to observe high-velocity hydrogen absorption features in GRB afterglows, such as described by Starling et al. (2005) on GRB 021004.

On the other hand, van Marle et al. (2005) argue that the presence of an intermediate velocity component in the afterglow of GRB 021004 implies that the WR phase was short, i.e. that the WR shell was still intact when the star exploded. This implies that the initial mass of the progenitor must have been small (i.e. about $25 M_{\odot}$ ), since the smaller the initial mass, the shorter the WR phase (see Fig. 9 in Meynet \& Maeder 2005). But such stars have a very short $\mathrm{H}$-free WC stage. This is possibly in contradiction with the requirement of no hydrogen left in the atmosphere (MacFadyen \& Woosley 1999).
It is interesting to note that theoretical models seem to produce two very different types of GRB progenitors. On one hand, Hirschi et al. (2005) show that single WO type stars are the best GRB candidates, while the presence of hydrogen in GRB afterglows and the results of van Marle et al. (2005) point toward lower mass WR stars. Could these latter stars still produce GRB if they belong to an interacting binary system, where the companion helps to remove the hydrogen layers? The question remains open.

\section{Conclusion}

We have presented new medium-resolution NTT-EMMI spectra of the LMC eclipsing Wolf-rayet binary BAT99-129. We have been able to separate the spectra and find a reliable orbital solution. We assign a spectral type WN3ha + O5V to the system. The $\mathrm{O}$ star seems to appear possibly oversynchronous with the orbital motion. We find that the WR star contains a detectable amount of hydrogen, seen in absorption in its spectrum. While its actual characteristics are shared with the eWNL stars seen in the SMC (Foellmi et al. 2003a; Foellmi 2004), the evolution that led to the present state is certainly much different. Interacting and binary models that could be applied to systems like BAT129 (hence also to V444 Cyg) would be of major interest.

BAT99-129 appears to be a very interesting piece of the puzzle of GRB progenitors. However, the most important questions remain: what are the kinds of the progenitors of the long soft GRB progenitors? Are they single or binary stars? Are they low mass or high mass WR stars? The presence of absorption lines in BAT129 proved to be of the greatest interest and further studies of GRB afterglows will certainly confirm the importance of these absorptions.

BAT129 is definitely a very interesting target for X-ray observations, considering the eclipsing nature of the system and the clear signs of wind-wind collision. Unfortunately, we found no XMM, ROSAT, nor Chandra observations of this system. Moreover, the eclipses deserve much more study. In particular, the eclipses can be used to probe the actual sizes, in physical units, of the line emitting regions. This is now possible thanks to the complete orbital solution. We obtained VLT FORS1 spectra for this purpose and their analysis will lead to a forthcoming paper.

All in all, BAT99-129 appears to be very similar to the well known WR binary in our Galaxy, V444 Cyg.

Acknowledgements. C.F. would like to warmly thank Olivier R. Hainaut for help. C.F. also thanks J.-C. Bouret for useful comments, and P. Gandhi for checking X-ray data availability. A.J.F.M. is grateful to NSERC (Canada) and FQRNT (Québec) for financial support. This paper utilizes public domain data originally obtained by the MACHO Project, whose work was performed under the joint auspices of the U.S. Department of Energy, National Nuclear Security Administration by the University of California, Lawrence Livermore National Laboratory under contract No. W-7405-Eng-48, the National Science Foundation through the Center for Particle Astrophysics of the University of California under cooperative agreement AST8809616, and the Mount Stromlo and Siding Spring Observatory, part of the Australian National University. IRAF is distributed by the 
National Optical Astronomy Observatories, which are operated by the Association of Universities for Research in Astronomy, Inc., under cooperative agreement with the U.S. National Science Foundation. This research has made use of the SIMBAD database, operated at CDS, Strasbourg, France. We thank the referee, A. M. Cherepashchuk, for constructive comments, and N. Langer for suggesting to compute the minimum period.

\section{References}

Alcock, C., Allsman, R. A., Alves, D. R., et al. 1999, PASP, 111, 1539 Bartzakos, P., Moffat, A. F. J., \& Niemela, V. S. 2001, MNRAS, 324, 18

Beals, C. S. 1944, MNRAS, 104, 205

Bertiau, F. C., \& Grobben, J. 1969, Ricerche Astron., 8, 1

Bonanos, A. Z., Stanek, K. Z., Udalski, A., et al. 2004, ApJ, 611, L33

Breysacher, J., Azzopardi, M., \& Testor, G. 1999, A\&AS, 137, 117

Cherepashchuk, A. M., Koenigsberger, G., Marchenko, S. V., \& Moffat, A. F. J. 1995, A\&A, 293, 142

Conti, P. S., \& Frost, S. A. 1977, ApJ, 212, 728

Conti, P. S., \& Morris, P. W. 1990, AJ, 99, 898

Crowther, P. A., Smith, L. J., Hillier, D. J., \& Schmutz, W. 1995, A\&A, 293, 427

Demers, H., Moffat, A. F. J., Marchenko, S. V., Gayley, K. G., \& Morel, T. 2002, ApJ, 577, 409

Eggleton, P. P. 1983, ApJ, 268, 368

Feitzinger, J. V., \& Isserstedt, J. 1983, A\&AS, 51, 505

Foellmi, C. 2004, A\&A, 416, 291

Foellmi, C., Moffat, A. F. J., \& Guerrero, M. A. 2003a, MNRAS, 338, 360

Foellmi, C., Moffat, A. F. J., \& Guerrero, M. A. 2003b, MNRAS, 338, 1025

Galama, T. J., Vreeswijk, P. M., van Paradijs, J., et al. 1998, Nature, 395,670

Hamann, W.-R., \& Koesterke, L. 2000, A\&A, 360, 647

Hamann, W.-R., Koesterke, L., \& Wessolowski, U. 1995, A\&A, 299, 151

Hirschi, R., Meynet, G., \& Maeder, A. 2005, ArXiv Astrophysics e-prints

Le Floc'h, E., Duc, P.-A., Mirabel, I. F., et al. 2003, A\&A, 400, 499

Lewis, D., Moffat, A. F. J., Matthews, J. M., Robert, C., \& Marchenko, S. V. 1993, ApJ, 405, 312
MacFadyen, A. I., \& Woosley, S. E. 1999, ApJ, 524, 262

Marchenko, S. V., Moffat, A. F. J., \& Koenigsberger, G. 1994, ApJ, 422, 810

Marchenko, S. V., Moffat, A. F. J., Eenens, P. R. J., Hill, G. M., \& Grandchamps, A. 1995, ApJ, 450, 811

Marchenko, S. V., Moffat, A. F. J., \& Eenens, P. R. J. 1998, PASP, 110 1416

Marchenko, S. V., Moffat, A. F. J., Crowther, P. A., et al. 2004, MNRAS, 353, 153

Martins, F., Schaerer, D., \& Hillier, D. J. 2005, A\&A, 436, 1049

Massey, P. 1981, ApJ, 244, 157

Massey, P. 2003, ARA\&A, 41, 15

Massey, P., Puls, J., Pauldrach, A. W. A., et al. 2005, ApJ, 627, 477

Mathys, G. 1988, A\&AS, 76, 427

Meynet, G., \& Maeder, A. 2005, A\&A, 429, 581

Nugis, T., \& Niedzielski, A. 1995, A\&A, 300, 237

Petrovic, J., Langer, N., \& van der Hucht, K. A. 2005a, A\&A, 435, 1013

Petrovic, J., Langer, N., Yoon, S.-C., \& Heger, A. 2005b, A\&A, 435, 247

Postnov, K. A., \& Cherepashchuk, A. M. 2001, Astron. Rep., 45, 517

Prochaska, J. X., Bloom, J. S., Chen, H.-W., et al. 2004, ApJ, 611, 200

Rauw, G., Crowther, P. A., De Becker, M., et al. 2005, A\&A, 432, 985

Rigon, L., Turatto, M., Benetti, S., et al. 2003, MNRAS, 340, 191

Rousseau, J., Martin, N., Prevot, L., et al. 1978, A\&AS, 31, 243

Smith, L. F. 1968, MNRAS, 138, 109

Smith, L. F., \& Maeder, A. 1998, A\&A, 334, 845

Smith, L. F., Shara, M. M., \& Moffat, A. F. J. 1996, MNRAS, 281, 163

Starling, R. L. C., Wijers, R. A. M. J., Hughes, M. A., et al. 2005, MNRAS, 360, 305

Tutukov, A. V., \& Cherepashchuk, A. M. 2004, Astron. Rep., 48, 39 van den Bergh, S. 2000, PASP, 112, 529

van der Hucht, K. A. 2001, New Astron. Rev., 45, 135

van Marle, A.-J., Langer, N., \& Garcia-Segura, G. 2005, ArXiv Astrophysics e-prints

Vink, J. S., \& de Koter, A. 2005, ArXiv Astrophysics e-prints

Walborn, N. R., \& Fitzpatrick, E. L. 1990, PASP, 102, 379

Wellstein, S., \& Langer, N. 1999, A\&A, 350, 148

Wellstein, S., Langer, N., \& Braun, H. 2001, A\&A, 369, 939

Wyrzykowski, L., Udalski, A., Kubiak, M., et al. 2003, Acta Astron., 53,1 